\title{
Experimental Study of the Effect of Automated Fiber Placement Induced Defects on Performance of Composite Laminates
}

Kaven Croft ${ }^{a}$, Larry Lessard ${ }^{a,{ }^{*}}$, Damiano Pasini ${ }^{a}$, Mehdi Hojjati ${ }^{b}$, Jihua Chen ${ }^{b}$, and Ali Yousefpour ${ }^{\mathrm{b}}$

a Department of Mechanical Engineering, McGill University, 845 Sherbrooke Street West Montréal, Québec, Canada, H3A 2T5

${ }^{\mathrm{b}}$ National Research Council Canada, Institute for Aerospace Research, Aerospace Manufacturing Technology Center, 5145 Decelles Avenue Montreal, Quebec, Canada, H3T 2B2

* Corresponding author. E-mail address: larry.lessard@mcgill.ca, (514) 398-6305

\section{Abstract}

The Automated Fiber placement (AFP) process shows great potential for efficient manufacturing of large composite structures. However, uncertainties exist on mechanical performance of final product that are associated with the process induced defects. This experimental work investigates the effect of four principal defect types, namely gap, overlap, half gap/overlap and twisted tow on the ultimate strengths. Tests are executed at the lamina level (fiber tension, fiber compression and in-plane shear), as well as at the laminate level (open hole tension and open hole compression). Then each test is compared with a baseline configuration exempt from defects. Tests have revealed the minimal effects of a single and isolated defects on mechanical performance especially at the lamina level (around 5\%) compared to the laminate level (up to 13\%).

\section{Keywords:}
A. Tow
B. Defects
C. Mechanical Properties 


\section{Mechanical Testing}

\section{Introduction}

Composite materials are now increasingly used as large and complex primary structures in the aeronautics industry, because of their advantages over traditional materials. However, their well known manufacturing weaknesses drive many companies to develop new methods to obtain better part quality. The recent introduction of airplanes with parts made by the Automated Fiber Placement (AFP) process has increased the demand for this method. An AFP machine consists of a computer controlled robotic arm with a placement head end effector that lays bands of prepreg strips onto a mould in order to construct the layup. The bands are made with 8 to 32 prepreg strips, called tows, which are aligned side-by-side by the placement head. For each ply, the machine accurately places the bands on the mould respecting the proper ply angles and the covering technique. The part is then placed in an autoclave to polymerize the resin material and consolidate the plies. Another advantage of this process is the possibility of making variable stiffness laminates with curvilinear fiber path for the purpose of optimizing the composite structure. However, in order to manufacture complex shapes or variable stiffness parts, misalignments are induced on the band edges, which introduce gaps and/or overlaps. To overcome this situation, the software provided with the machine includes a cut-and-restart option that gives different approaches to limit these gaps and/or overlaps. Depending on the strategy chosen during the path generation, the machine can cut individual tows to create full gaps, full overlaps or a ratio of full gaps and full overlaps versus the initial defects. This option gives more control on the type of defect and on its size. In addition, the material and machine tolerances induce small random gaps and overlaps within the entire structure that cannot be removed, because they are an integral part of the processing. Furthermore, 
missing, twisted, or spliced tows are sometimes laid down during manufacturing, which create uncertainties and must be repaired during the process. Several researchers have established methods to reduce the effects of these defects on a composite structure. Some examples are: the staggering technique [1] and placement of $\pm 45^{\circ}$ layers on the top and bottom layers [2]. However, even with these techniques, the defects are still present and their influence on the performance of the structures are not well understood.

Over the years, research has demonstrated the feasibility of using the AFP process by testing panels and aerospace parts. From these studies it can be concluded that, overall, the parts perform similarly or better than those made by hand lay-up [3-5]. In a case study, Measom and Sewell [3] demonstrated the effect of the tow width $(12.7 \mathrm{~mm}$ [0.5"], $19.1 \mathrm{~mm}$ [0.75"] and $25.4 \mathrm{~mm} \mathrm{[1"])} \mathrm{and} \mathrm{the} \mathrm{cut-and-restart} \mathrm{option,} \mathrm{both} \mathrm{on} \mathrm{the} \mathrm{interlaminar} \mathrm{shear}$ performance. They revealed that the size of the bandwidth had minor effects on the interlaminar shear tests, which confirms the good consolidation obtained at the edges. Also, the gaps-fiber-placed configuration had equivalent strength to the hand layup tape, which draws attention to the small effect of the gap type of defect on the structural performance. These results confirmed the potential of this technology; however, more recent machines now use smaller tow width tape of width $3.2 \mathrm{~mm}$ [0.125"] or $6.35 \mathrm{~mm}$ [0.25”].

Sawicki and Minguet [1] explored the effect of a gap width $(0.76 \mathrm{~mm}$ [0.03"] and $2.5 \mathrm{~mm}$ [0.10"]) by means of the compression strength test (notched and unnotched specimens). It is concluded that any gap size induced a strength decrease. It is shown that the rate of this decrease is pronounced for gap sizes smaller than $0.76 \mathrm{~mm}[0.03$ "] and the strength reduction is relatively constant for larger gap sizes. They found that the compression strength reduction for unnotched and notched laminates was the same. The main failure 
mechanism for specimens in compression was caused by the out-of-plane waviness induced by the defects on the subsequent plies.

More recently, Turoski [6] conducted an experimental and numerical analysis to obtain the effect of the number of gaps for several mechanical properties, such as tension and compression for notched and unnotched quasi-isotropic laminates. It is concluded that in general, the unnotched specimens are more influenced by the defects than the notched specimens. This indicates that the stress concentration associated to the notch induces more strength reduction than the defects. Moreover, the amount of defects on the unnotched specimens is related to the strength reduction and confirms the performance reduction of gaps on structures. Overall, this recent research has given a small overview of the failure mechanisms induced by the gap type of defect; however, other types of defects have not been investigated experimentally and most of the results have been obtained by means of numerical analysis.

The lack of results from previous research creates uncertainties which justify the need to understand the real effects of the main defects. For this reason, the purpose of this investigation is to quantify the effect of the manufacturing induced defects by testing four principal types of defects, namely gap, overlap, half gap/overlap and twisted tow. To do so, all these defects have been tested at three lamina levels (fiber tension, fiber compression and in-plane shear) and two laminate levels (open hole tension and open hole compression). Experimental results have been compared with a baseline configuration without defects. To quantify the effect of defects on ultimate strength, a high toughness autoclave cure carbon-epoxy prepreg have been used.

\section{Defect description}


During the manufacturing, several defects appear randomly and simultaneously; thus, it is important to know the combined effects of the different type of defects on a real and complex structure. In addition, the loading conditions and the part geometry change the effects of the defects, which add more complexity to the analysis; however, the understanding of all possible behaviours for each defect would require numerous experiments and configurations. For this reason, the investigation of each individual defect would provide a trend that will help to isolate the critical parameters, to evaluate the integrity of the laminate and to understand the need of repairing the process induced defects such as missing tows or twisted tows. Then, it may be possible to extend the understanding to a real case and combine the effects.

Each defect type creates thickness build-up that influences the in-plane extension and shear stiffness coupling terms in the ABD matrix (from classical laminate theory). In order to minimize the internal disturbance caused by these terms, each defect has been placed in the middle layers of a symmetric and balanced laminate, and this position has been kept constant throughout all the experiments. Also, it is assumed that one tow defect generates a small effect on a structure because usually the material used in the AFP process is thin and tough. Consequently, to increase the effect and to be able to capture the variability between configurations, the size of the defect has been defined as one tow width and two tows thick. In other words, to increase the defect area, the two middle layers have been modified.

The theoretical configurations used are illustrated in Figure 1 for all four defect configurations. For instance, the gap configuration (Figure 1a) is made by removing two tows one on top of each other. Like the previous configuration, the overlap (Figure $1 \mathrm{~b}$ ) is created by adding two tows instead of removing them. On the other hand, the half 
gap/overlap (Figure 1c) is created by removing two tows and by shifting them by half a tow. The twisted tow (Figure 1d) configuration is created by flipping the tow upside down as illustrated on the figure. Furthermore, only one tow is modified for this last configuration because of the difficulty to accurately place two defects one on top of each other and because two defects of this nature rarely occur at the same location.

Then, the orientation of the defects is defined according to the middle layers of the laminate used for each test. In order to obtain the worst-case scenario for each of them, the defect orientation is defined according to the assumption about their failure mechanisms. Thus, the details of each orientation and layup will be described in more detail in the following section.

\section{Tests and coupons description}

With the intention of obtaining a large range of effects, tests are executed under both lamina (fiber tension, fiber compression and in-plane shear) and laminate (open hole tension and open hole compression) levels. These properties are essential to understand the behaviour of a real and complex structure under multidirectional loads. The lamina properties are associated with the ply and are used during the layer definition, while the laminate properties are associated with the laminate performance and are used to define the failure criterion. Diagrams in Figure 2 show the different coupons for each test with the defect location designated by plane lines in the test section. For each test, a panel is made

and the defects are manually inserted during the manufacturing. Then, the location of each defect is marked on the top of the laminate, with a special marker that does not affect the laminate properties, in order to accurately place the defect on the test section of the specimen. 
The fiber tension test (Figure 2a) is designed with respect to the ASTM 3039 Standard [7], which gives the guidelines to create the geometry and the execution of the test. To be consistent with the lamina level, a laminate with eight $0^{\circ}$ plies is created to make the stresses equal inside each layer. To be consistent with the fiber orientation, the defect is placed along the length and is located in the middle of the width to eliminate the edge effects. Also, E-glass tabs have been glued at the extremities to suppress the laminate from splitting inside the gripping area.

The fiber compression test (Figure 2b) is designed according to the ASTM 3410 standard [8]. For the same issues that govern the previous test, the laminate is made of $0^{\circ}$ plies with the defect placed in the middle of the width and along the entire length $\left(0^{\circ}\right)$. The main consideration with this test is the buckling of the test section that considerably reduces the ultimate strength; therefore, the thickness of the laminate has been increased to 16 plies and the gage length has been reduced to avoid this instability.

The in-plane shear properties are the most difficult test to execute because of the nonuniform and non-linear stress field inside the test section. Consequently, many types of shear tests have been developed over the years. For this investigation, the V-Notched Beam Method or losipescu Test (ASTM 5379 standard [9]) has been chosen, primarily due to its small specimen size and its simplicity. However, additional attention was considered to shape the specimen notches without inducing delamination. To obtain a pure shear mode inside the test section, this method requires a $0-90^{\circ}$ layup that is used for both 16 plies specimen configurations (Figure 2c): defect along the length $\left(0^{\circ}\right)$ and defect along the width $\left(90^{\circ}\right)$ are induced. 
The Open Hole Tension (OHT) test is used to obtain the performance of a laminate subjected to a non-constrained hole, which creates a stress concentration that initiates the failure earlier. The ASTM 5766 standard [10] defines the quasi-isotropic layup ([45/0/$45 / 90]_{2 s}$ ), the geometry and the test definition. The standard suggests a minimum width-todiameter ratio of 6 , which was used for all open hole tests in order to eliminate the edge effects. Thus, the defect has been placed along the width $\left(90^{\circ}\right)$ and crosses the hole at its center (Figure 2d) where the stress concentration is maximized.

The Open Hole Compression $(\mathrm{OHC})$ test is used to obtain the same type of results as the OHT test, but in compression. This test is defined according the ASTM 6484 standard [11] and requires the Boeing open hole test fixture, which includes an anti-buckling device. The suggested quasi-isotropic layup $\left([45 /-45 / 90 / 0]_{2 s}\right)$ is slightly different than that of the OHT test but contains the same amount of each ply angle. As mentioned previously, the weakness and limitation of compression tests are usually caused by the buckling effect, thus the defect is located along the length to observe its effect (Figure 2e). Extra OHT samples have been modified in $\mathrm{OHC}$ samples to look at the effect of a defect along the width, which is located in the highest stress concentration area (Figure 2e). The difference in stacking sequence makes it impossible to compare the values of the ultimate strength but gives a good idea about the individual effect compared with its baseline configuration.

\section{Real defect geometry}

The assumption often used to define the defect geometry is that the area associated with the missing tow is totally filled by resin (resin rich area). Since the high pressure provided by the autoclave makes this assumption uncertain, micrographs of each investigated defect have been taken to reveal the real geometry. 
First, micrographs of the unidirectional laminates reveal that tolerance-induced defects happen repeatedly in all layers and can be situated one on top of the other, which contributes to creating variations in thickness. This variation is inversely proportional to the thickness of the laminate, because a thicker unidirectional laminate helps to hold the pressure of the autoclave. It was observed that tows have the ability to move according to the previous layers and fill all possible gaps, regardless of the size (Figure 3).

Consequently, for the unidirectional laminates, the gap type of defect is not composed of a resin rich area. The other kinds of defects are guided by the same rules and give good laminate integrity and consolidation. The conclusions drawn from the unidirectional laminates give a good idea about the reaction of each independent layer inside a nonunidirectional laminate.

At the laminate level, the previous conclusions are still applicable; however the fiber waviness of the other plies needs to be considered. Fiber waviness is mostly resulting from the pressure that pushes the other plies onto the defect. For example, the plies curve to fill a gap or to overpass an overlap region. Also, the radius of curvature of both gaps and overlaps are equal but on the opposite sides of the laminate. In addition, the fiber waviness is more concentrated on the top of the defect, due to the position of the mould and the bottom plies, which constrain its displacement. Furthermore, the fiber waviness decreases as it reaches the top layer because of the out-of-plane stiffness of the plies that hold the pressure non-evenly throughout the thickness. The main conclusion is that the defect size is more critical for laminate integrity because smaller defects yield smaller radii of curvature. For example, for the half gap/overlap configuration, the pressure was not sufficient to bend all the other plies inside the gap region. Consequently, a resin accumulation can be observed. Figure 4 clearly shows this last observation for a $0-90^{\circ}$ 
laminate (in-plane shear test) with a half gap/overlap type of defect. The comparison of the different quasi-isotropic layups (OHT and $\mathrm{OHC}$ tests) have shown that a smaller angle difference between two consecutive plies makes the bending of the tows easier within the defect, which reduces the resin rich area.

\section{Test results}

In order to obtain accurate and consistent results, statistical tools have been used throughout this investigation. For each type of test, an average of six specimens has been used to define the ultimate strength of each defect configuration. The coefficient of variation for each average has been calculated to represent the error. With these results, a bar chart has been constructed for the different tests summarizing the effects of the defect configurations compared with a baseline configuration without defect. Also, for each defect configuration, a statistical test on the average based on the p-value was executed. Based on the assumption of a $5 \%$ maximum variation of the ultimate strength, the p-value represents the probability to find a sample outside of this range. Consequently, the lower this value, the more accurate is the sampling and the more significant is the test. This value is directly related to the complexity and variability of the coupons parameters and of the type of defect. The following sections will present and analyse all results.

\subsection{Tension}

The fiber tension test was executed on a $100 \mathrm{kN}$ MTS test frame with hydraulic grips. All specimens have shown a catastrophic mode of failure on the entire test section, which can be seen in Figure 5 for a specimen without defect. This is the consequence of the toughened resin system, which efficiently transfers and accumulates the load, and creates a uniform stress distribution inside the entire test section. The small variation between the samples (Figure 6) illustrates the small effect of the different defect configurations with a maximum reduction of $3.4 \%$ for the half gap/overlap configuration. As shown in the micrographs, a unidirectional laminate helps to uniformly fill the defect, thus reducing its 
effect. However, the failure usually occurred next to the defect and then propagated along it. This was revealed by observations during the tests and by many broken specimens that show visible failure discontinuity on the edges of the defect. The decrease of the ultimate strength obtained for the gap configuration and the increase of the same property for the overlap configuration are slightly influenced by the error during the area calculation and due to the thickness variation. In addition, the small effect of these defects (less than $2 \%$ ) suggests that this error is less than $1 \%$ and can be considered negligible. It is also negligible for the half gap/overlap configuration due to the geometry of the defect itself. The tow edges for this last defect configuration are more problematic due to the staggering techniques used, which aligned the defect edges through the thickness with the next middle layers and, consequently, weakened the thin 8 ply laminate. In addition, this defect has a tendency to become straight (out-of-plane), during the load application, which induced a force through the thickness and initiated cracks along the defect. Another interesting conclusion for this test is the increase of ultimate strength for the twisted tow configuration. More investigation, especially looking at the stress-strain field, is required to fully understand and explain this phenomenon. Overall, the results show the relatively negligible effect of the different defect configurations and a good integrity of the laminate. Also, the $0.5 \%$ maximum overall $p$-value suggests consistent and representative results for all test configurations.

\subsection{Compression}

The compression test was executed on a $250 \mathrm{kN}$ MTS test frame with an IITRI compression fixture. Samples were usually broken inside either the test section or on the tab edges. Failure mainly occurred at $45^{\circ}$ through the thickness and only one sample showed a brooming failure in the test section, which are both defined acceptable by the 
standard. Figure 7 illustrates the typical mode of failure observed for a specimen without defect. Similar to the previous test, the defects were smoothed by the unidirectional layup and the laminate shows less thickness variation due to the increase of layers, as depicted in the micrographs. The results in Figure 8 show the negligible effect of the different defects with a maximum reduction of $1 \%$ of the ultimate strength. The large coefficient of variation (error bar) makes it difficult to capture a pattern between the different defect configurations; thus, the main conclusion is that all these types of defect have negligible effect at the lamina level. Also, similar to the tension test, the increase of the overlap configuration strength is mainly due to the error associated with the area calculation. Overall, the p-values show insignificant variation (less than $2 \%$ ) except for the gap $(21 \%)$ and the twisted tow (9\%) configurations.

\subsection{In-plane shear}

The V-Notched in-plane shear test was executed on a $5 \mathrm{kN}$ MTS test frame with the recommended fixture. The two different defect configurations failed with the same mechanism as that of a typical $0-90^{\circ}$ layup and was defined acceptable by the standard. This failure is represented by the fiber shifting in the $0^{\circ}$ plies and a matrix failure in the $90^{\circ}$ plies. It was possible to confirm this type of failure by a visual inspection of the specimens (Figure 9). This type of test creates a non-constant stress-displacement curve that is characteristic of this $0-90^{\circ}$ layup and creates a pure shear state in the test section. Consequently, the complex behaviour of the stress-displacement curve makes this test more difficult to interpret. At the beginning, the modulus is high and then suddenly reduces. This second modulus stays almost constant up to the first stress drop, which is how ultimate strength is defined. Afterwards, the material yields more under load until there is no more loading capability or, in some cases, the fixture reaches its displacement 
limits. For each sample, the ultimate strength has been found from this curve. However, the load drop did not occur for all samples due to the high toughness of the resin, which was confirmed by the large displacement of the tested specimens' ends (around $3.5 \mathrm{~mm}$ displacement). In such cases, the standard assumes the definition of the ultimate strength at the $5 \%$ strain level. For tough resin systems, this criterion is imprecise and, consequently, these few cases were not considered. Nevertheless, enough specimens were successfully tested to obtain good averages and precision, which was confirmed by the small coefficient of variation obtained. The p-value analysis, despite the small sampling, had scatter with values less than $5 \%$, except for four configurations that give a maximum of $17 \%$ (gap and overlap configurations along the width; and overlap and half gap/overlap configurations along the length). These high $p$-value uncertainties are the consequence of the complexity of this test (machining, defect localization and the type of defect) and the small test section. The results were then compared for both defect orientations (Figure 10) and it can be seen that the defect along the width has more effect than the defect along the length with a maximum reduction of $12 \%$ (overlap) compared to $9 \%$ (half gap/overlap) reduction. As a beam method, the $0^{\circ}$ layers hold more load than the $90^{\circ}$ layer because of the different failure mechanism. On the other hand, a defect along the width $\left(90^{\circ}\right)$ creates area where crack can more easily propagate (matrix cracking). This defect orientation also induces fiber waviness in the $0^{\circ}$ layers, which considerably reduces the strength of these layers. The overview of all results shows that the defect orientation largely influences this property but, overall, the reduction is small (less than $5 \%$ ).

\subsection{Open Hole Tension (OHT)}

The OHT test was executed on a $100 \mathrm{kN}$ MTS test frame similar to the uniaxial tension test. For all samples, the failures were initiated at the edge of the hole along the width (at 
the highest stress concentration area) and then have propagated to the weakest plies i.e. $90^{\circ}$ and $\pm 45^{\circ}$. This mode of failure is shown in Figure 11 for a specimen without defect. During this test, the stress-displacement curve looks comparable to the tension test, however, with smaller strength and the end of the curve is also more jagged. The small scale of the graph (Figure 12) shows the negligible influence of the different defect configurations with a maximum overall reduction of $1 \%$ (gap). The accuracy of these results are confirmed by the low p-value (nearly $0 \%$ ) for every specimen. This observation suggests that the defects have only a minor effect compared with the stress concentration factor created by the non-constrained hole. The results of this test support the findings of Turoski [6] and confirms that the defects should have more effect on the performance of the unnotched laminates.

\subsection{Open Hole Compression (OHC)}

The $\mathrm{OHC}$ test was executed on a $250 \mathrm{kN}$ MTS test frame with the Boeing open hole fixture. Similar to the previous test, failure was triggered by the stress concentration and by the weakest plies, which in this case are the $0^{\circ}$ and $\pm 45^{\circ}$ plies. During this test, the gage section cannot buckle, because it is supported by the configuration of the fixture. In contrast, the micro-buckling of the fibers, especially around the hole, makes the $0^{\circ}$ plies critical and, for this reason, two defect orientations have been tested, i.e., a defect along the length and another along the width. All samples have shown a toughening mechanism that is characterized by a micro-buckling of the $0^{\circ}$ plies in the vicinity of the hole (maximum stress concentration area) and followed by the delamination of the next layers. This delamination propagates all around the hole and consequently the ultimate strength is related to the delamination size [12]. Figure 13 illustrates, for a specimen without defect, the crack propagation that occurs along the width of the weakest plies and the 
delamination that propagates at the hole edge that is responsible for the toughness mechanism. The summary of the results (Figure 14) illustrates the improving behaviour for all types of defect along the length. Any defect along the length creates a weak region on the edge of the hole at $0^{\circ}$, which increases the stress at this location during the loading and locally facilitates delamination. However, the failure is still initiated along the width but the propagation around the hole is facilitated by the defect (and depends on the type of defect). Consequently, the strength increase is more or less influenced by the total delamination area in the vicinity of the hole (causing a softening of the local stress concentrations). This toughness phenomenon improves the ultimate strength (maximum of $13 \%$ ), but also increases the damage area inside the laminate. On the other hand, every configuration of defect along the width increases the stress concentration at the critical position, which reduces the properties. Width defects induce fiber waviness in the $0^{\circ}$ layers, which are more inclined to fail by micro-buckling. Consequently, the radius of curvature of the different defect configurations influences the ultimate strength. As the gap and overlap configuration have almost the same radius of curvature, the strength is equal (9\% reduction). The small radius of curvature of the half gap/overlap configuration creates the highest decrease $(12 \%)$. The consistency of these results is confirmed by the low $p$ values (less than 1\%), except for the half gap/overlap configurations (15\% for the defect along the length and $18 \%$ along the width) and for the gap configuration ( $7.5 \%$ along the length). Any small variation of the defect geometry and location of the defect relative to the hole influences the variability between samples, thus the p-value. Consequently, complex defect configurations have more variability, as is the case for the half gap/overlap configuration. Another interesting observation is the difference between the two baseline configurations, which were made with different quasi-isotropic layups. These laminates had the same amount of each ply angle, but a different ply distribution inside the 
laminates, which influenced the strength by about $7 \%$. The OHT layup was made using a smaller angle difference between two consecutive plies (fine ply distribution) and, consequently, created lower terms in the torsion coupling matrix $D_{\text {is }}[13]$, resulting in lower interlaminar shear and a higher ultimate strength.

\section{Conclusion}

The test results give a general overview of the influence of defects on laminate behaviour under several kinds of loadings and laminate configurations. The main conclusion is that the ultimate strength is less affected by the different defect configurations at the lamina level (overall less than $5 \%$ ) as opposed to the laminate level (up to $13 \%$ ). This was confirmed by the micrographs that show good integrity for the unidirectional laminate and, consequently, each individual ply in a laminate has appropriate consolidation. On the other hand, laminates with varying plies angles introduce fiber waviness around the defects. This fiber waviness is influenced by several variables (plies angles, defect geometry, position and pressure) that modifies the final geometry of the defect and, consequently, mainly affects the compression properties. For this reason, the in-plane shear tests have demonstrated a larger effect (12\%) when the defect is placed along the width, due to the induced fiber waviness in the $0^{\circ}$ layers. At the laminate level, the results obtained during the OHT test confirmed the near-negligible effect of defects because these tests are likely dominated by the stress concentration; however, the $\mathrm{OHC}$ tests have demonstrated the large influence of the defect orientation on the ultimate strength for tough materials because of the fiber micro-buckling mode of failure. A defect along the length increases the stress concentration at $0^{\circ}$, which generates a more uniform stress concentration around the hole and increases the strength. On the other hand, a defect along the width increases the stress concentration where it is already the highest $\left(90^{\circ}\right)$ and, consequently, 
significantly reduces the ultimate strength. Table 1 summarizes all previous results by giving the trend of each defect configuration for every test. A variation of $\pm 3 \%$ is defined to be negligible and is represented by a black horizontal line. The arrows represent an increase or a decrease of more than $3 \%$. This table shows that usually a defect that gives an improvement in one mechanical property also penalizes another property; therefore, for a real part with multidirectional loads, it is difficult to gauge the advantages of these increases. In addition, as is the case for the $\mathrm{OHC}$ test, the improvement is sometimes the consequence of a damage accumulation process (delamination), which deteriorates the integrity of the laminate and may need to be repaired. In conclusion, the results observed during many tests suggest that multiple defects should be considered in future tests and, consequently, the defect distribution will certainly become an important variable. This investigation should help to define a qualification process, to understand the effect of a single defect and help giving a method to predict the performance of composite structures considering the manufacturing defects.

\section{Future Work}

Compared with aerospace structures that are more complex and support multi-directional loadings, the investigated defects represent the lowest level of effect because of the amount and the definition of the defect used. The influence of the defect parameters on the part geometry is difficult to predict, thus, combinations of manufacturing defects should be considered to fully explore all cases and be able to optimize the design. For all properties, different parameters should be addressed, such as; the tow width; defect parameters and defect distribution; processing variables and the type of laminate. Consequently, the complexity of parts manufactured with the AFP process will require a 
larger test plan to fully capture the failure mechanism of the defects and to evaluate them in aerospace structures.

\section{Acknowledgements}

This work was financially supported by the National Research Council of Canada (Aerospace Manufacturing Technology Centre, Institute for Aerospace Research). 


\section{References}

1. Sawicki A.J. and P.J. Minguet. The Effect of Intraply Overlaps and Gaps Upon the Compression Strength of Composite Laminates. 39th AIAA Structural, Dynamics, \& Materials Conferences. Long Beach, CA. 1998. p. 744-754.

2. larve E.V. and R. Kim, Strength Prediction and Measurement in Model Multilayered Discontinuous Tow Reinforced Composites, Journal of Composite Materials, 2004; 38(1), p. 5-18.

3. Measom R. and K. Sewell. Fiber Placement Low-Cost Production for Complex Composite Structures. American Helicopter Society 52nd Annual Forum. Washington, DC. 1996. p. 12.

4. McManus H.L. and Y.-P. Mak. Strain Rate and Manufacturing Technique Effects on the Damage Tolerance of Composite Laminates. Structural Dynamics and Materials Conference. La Jolla, CA: AIAA/ASME/ASCE/AHS/ASC Structures. 1993. p. 853-1862.

5. Sawicki A.J., et al. Structural Qualification of V-22 EMD Tow-Placed Aft Fuselage. American Helicopter Society 51st Annual Forum. 1995. p. 1641-1653.

6. Turoski L.E., Effects of Manufacturing Defects on the Strength of Toughened Carbon/Epoxy Prepreg Composites, Mechanical Engineering, 2000, Montana State University, Bozeman, p. 125.

7. American Society for Testing and Materials, ASTM D3039/D3039M, Standard Test method for Tensile Properties of Polymer Matrix Composite Materials, 2006, Philadelphia.

8. American Society for Testing and Materials, ASTM D3410/D3410M, Standard Test method for Compressive Properties of Polymer Matrix Composite Materials with Unsupported Gage Section by Shear Loading, 2006, Philadelphia.

9. American Society for Testing and Materials, ASTM D5379/D5379M, Standard Test method for Shear Properties of Composite Materials by the V-Notched Beam Method, 2006, Philadelphia.

10. American Society for Testing and Materials, ASTM D5766/D5766M, Standard Test method for Open Hole Tensile Strength of Polymer Matrix Composite Laminates, 2006, Philadelphia.

11. American Society for Testing and Materials, ASTM D6484/D6484M, Standard Test method for Open Hole Compressive Strength of Polymer Matrix Composite Laminates, 2006, Philadelphia. 
12. Suemasu H., H. Takahashi, and T. Ishikawa, On failure mechanisms of composite laminates with an open hole subjected to compressive load, Composites Science and Technologiy, 2006(66), p. 634-641.

13. Daniel I.M. and O. Ishai, Engineering Mechanics of Composite Materials, 2 ed, Oxford University Press, 2005. 


\section{Figure Captions}

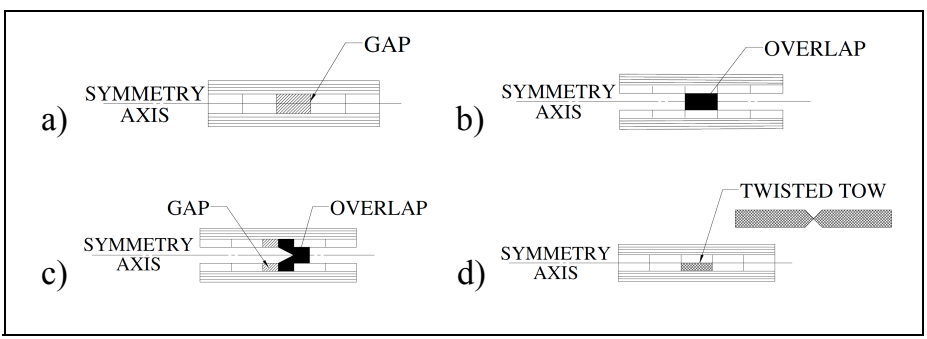

Figure 1: Defect configuration; a) gap; b) overlap; c) half gap/overlap; d) twisted tow

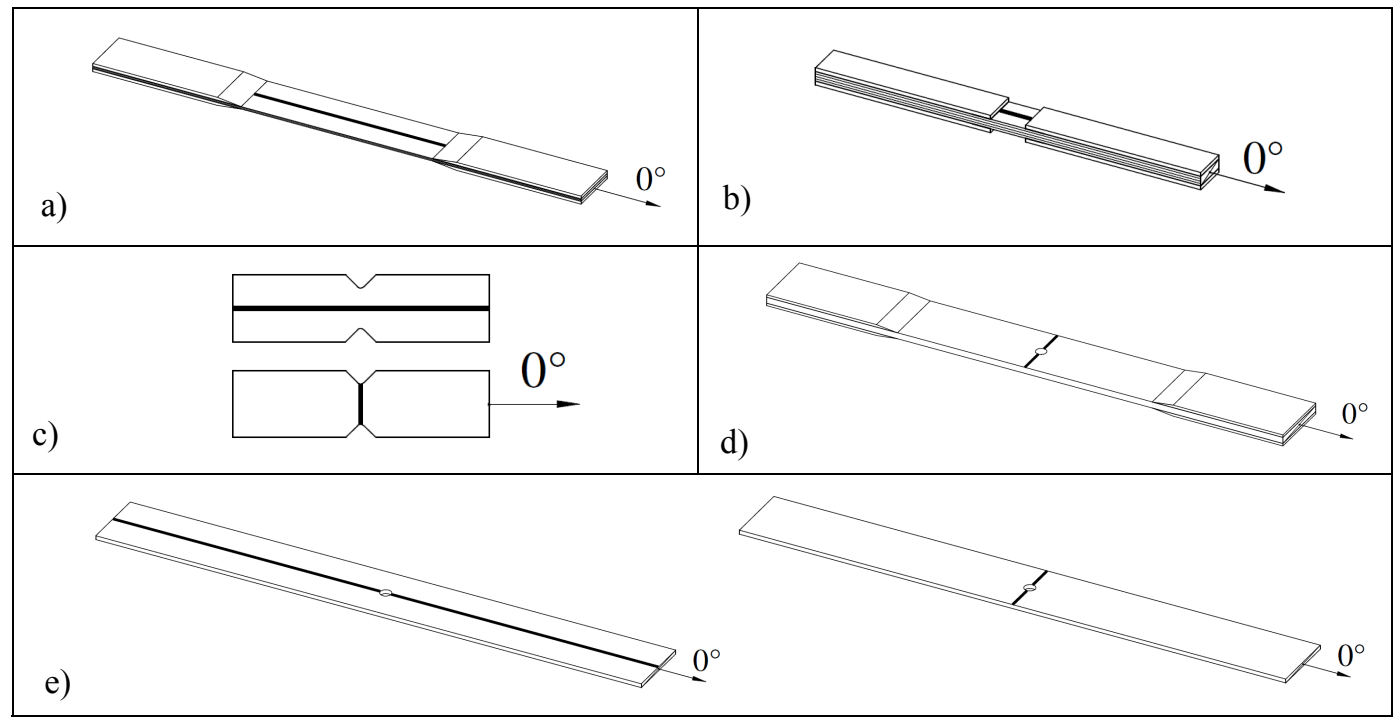

Figure 2: Defect orientation; a) fiber tension test; b) fiber compression test; c) in-plane shear test; d) OHT; e) OHC 


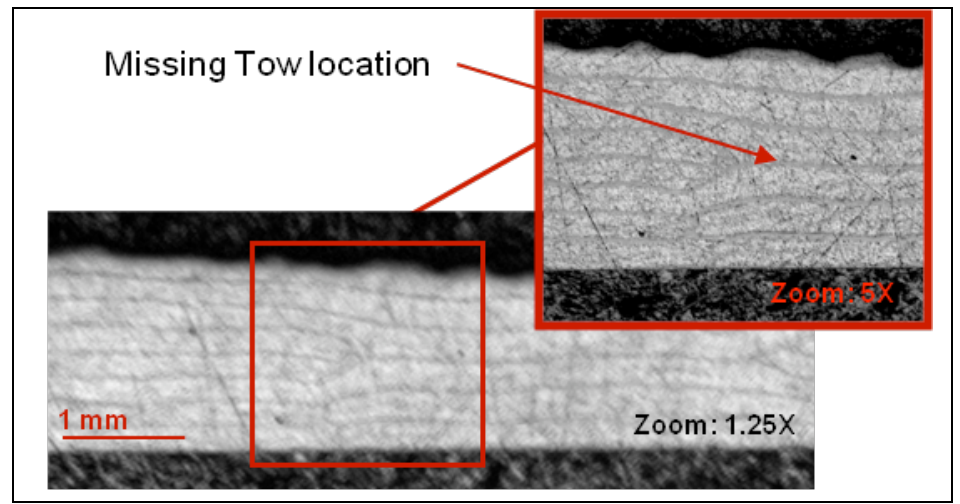

Figure 3: Tension gap specimen (magnification 1.25X and $5 X)$

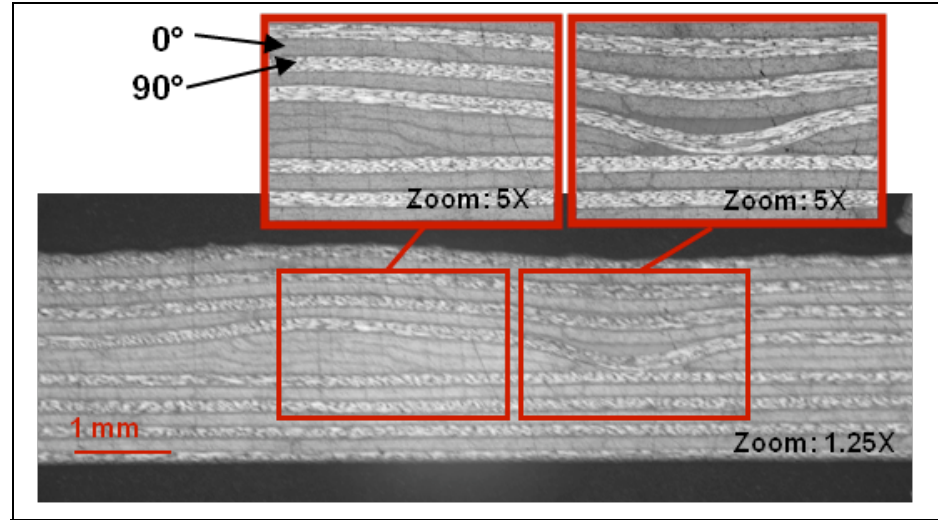

Figure 4: In-Plane shear specimen with half gap/overlap (magnification 1.25X and 5X)

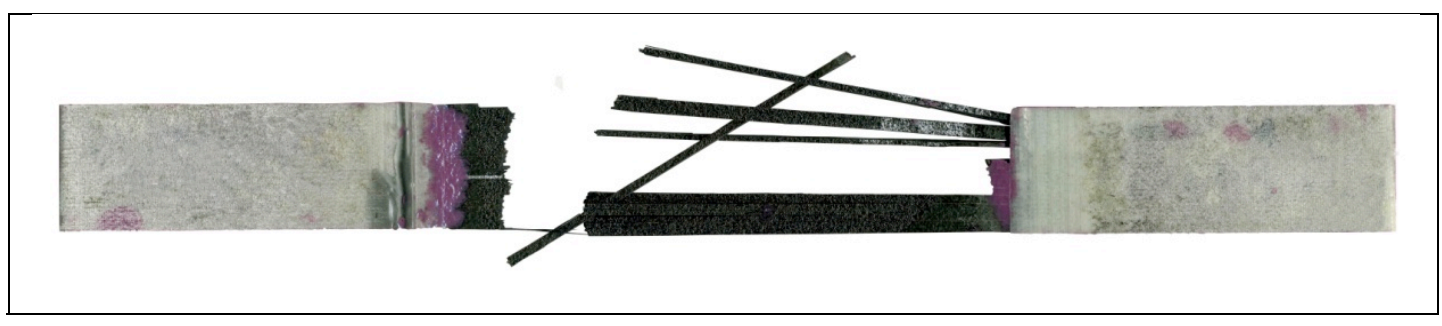

Figure 5: Typical Tensile failure for a coupon without defect 


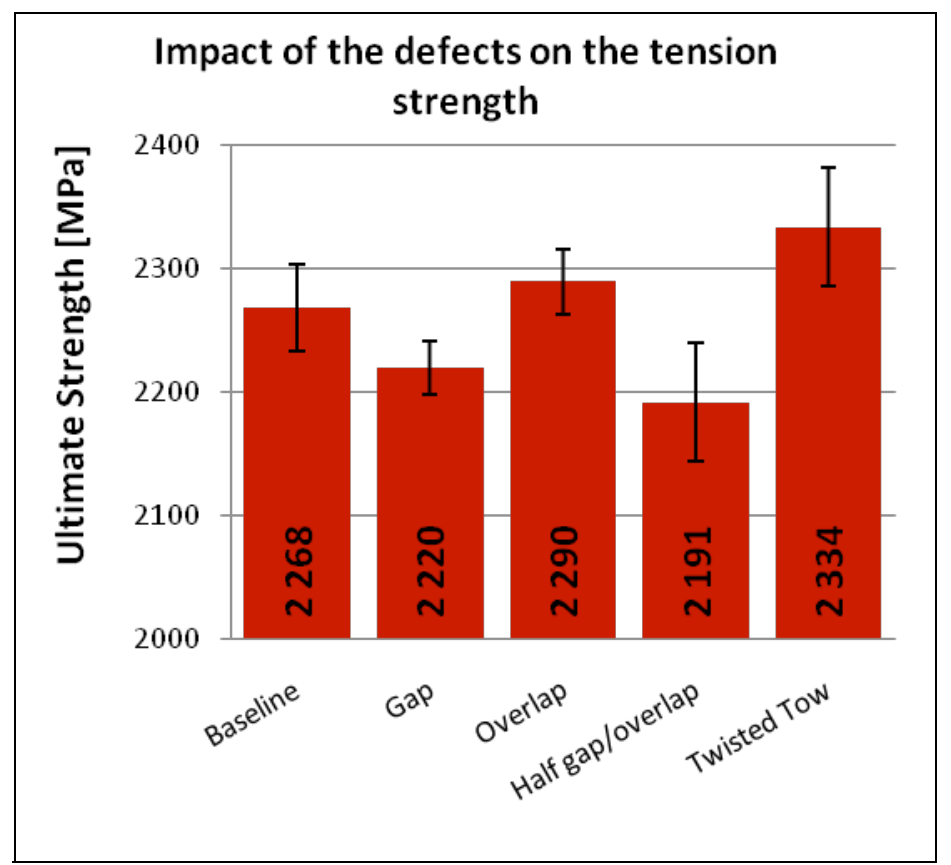

Figure 6: Tensile strength variation as a function of the type of defect

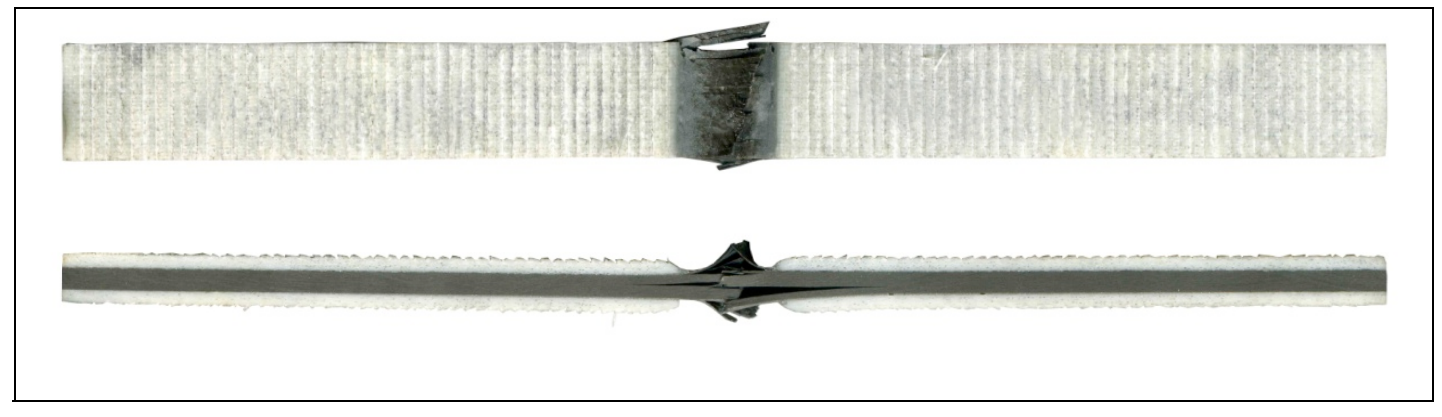

Figure 7: Typical Compression failure for a coupon without defect 


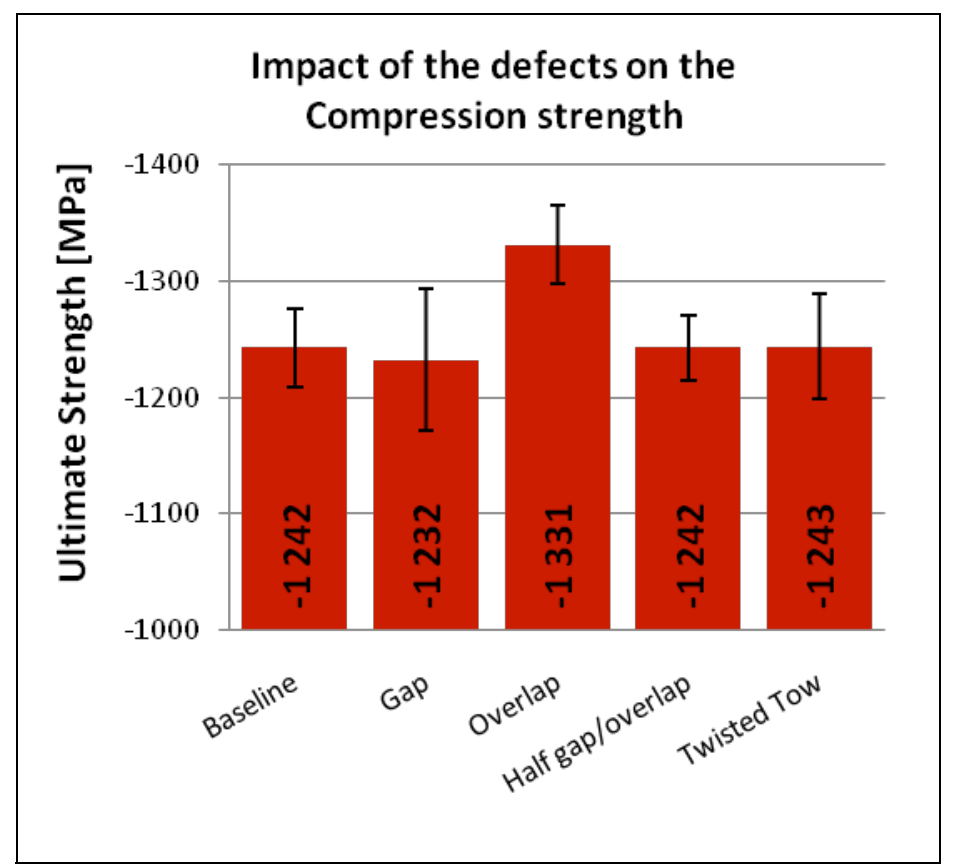

Figure 8: Compression strength variation as a function of the type of defect

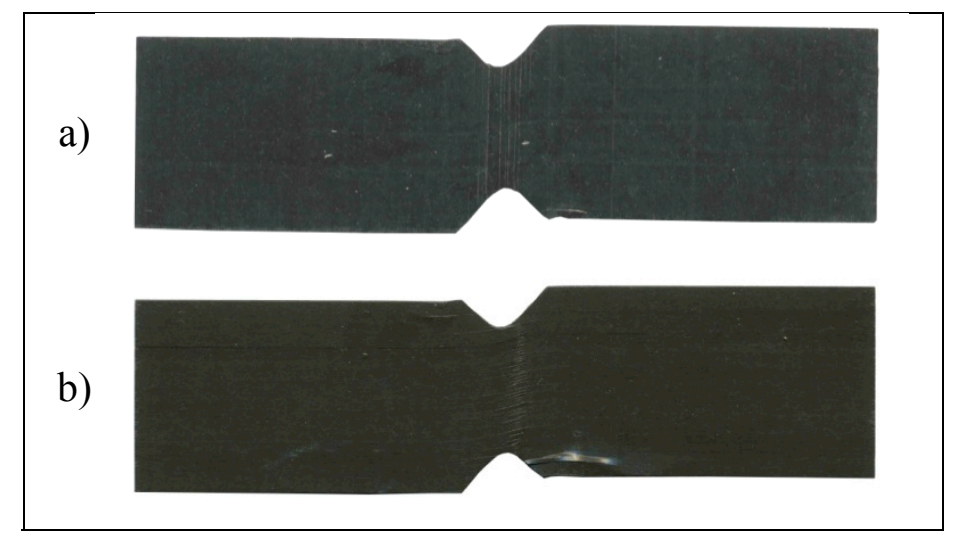

Figure 9: Typical V-Notched failure for a coupon without defect; a) defect through the length; b) defect through the width 


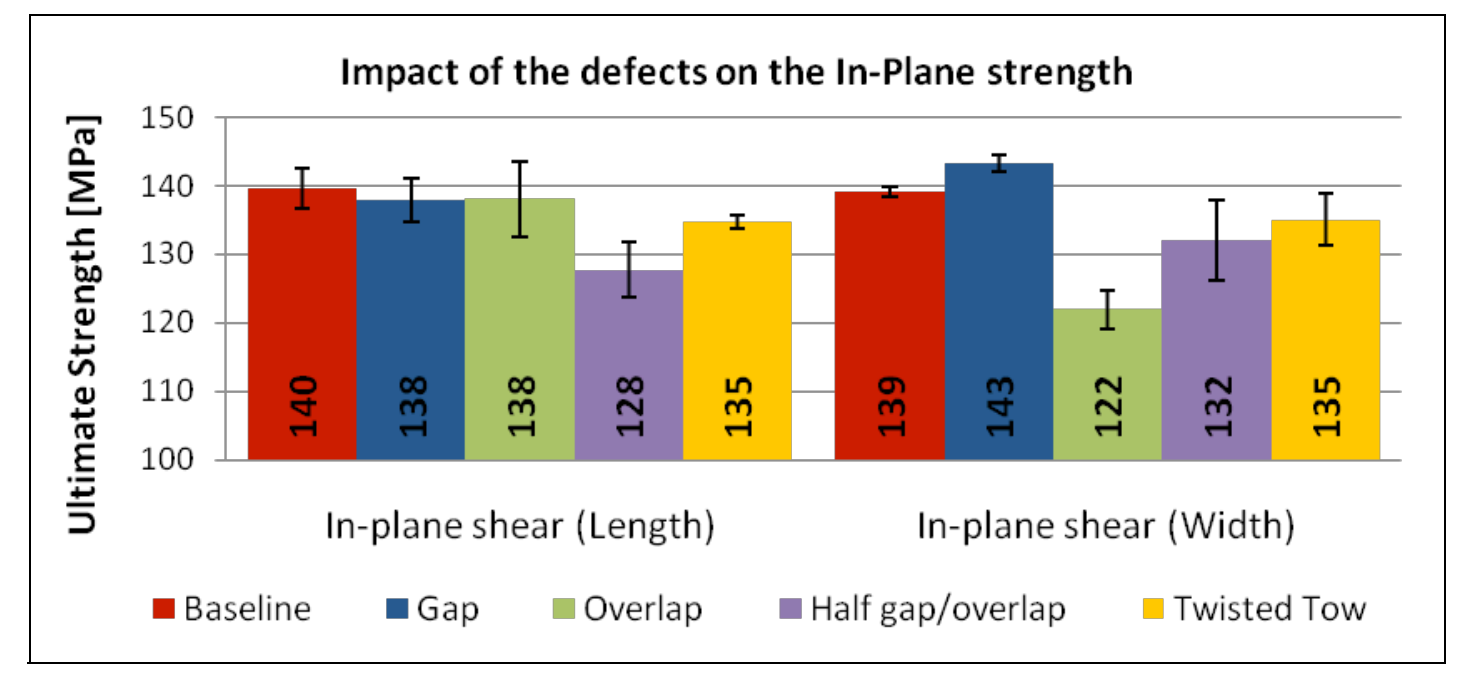

Figure 10: In-plane shear strength variation as a function of the type of defect

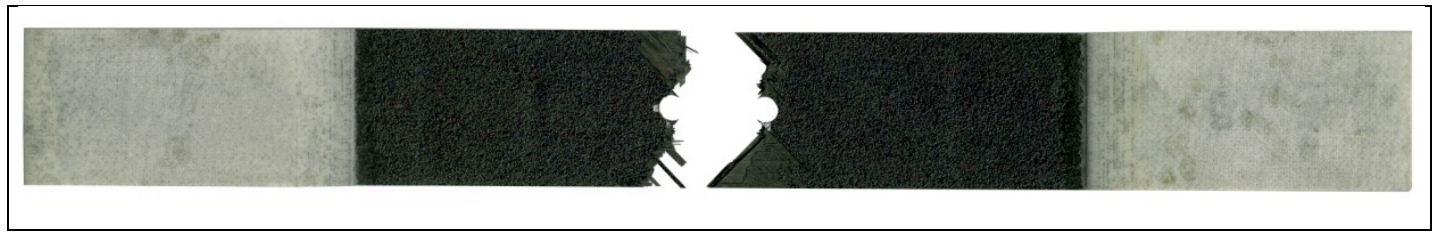

Figure 11: Typical OHT failure for a coupon without defect

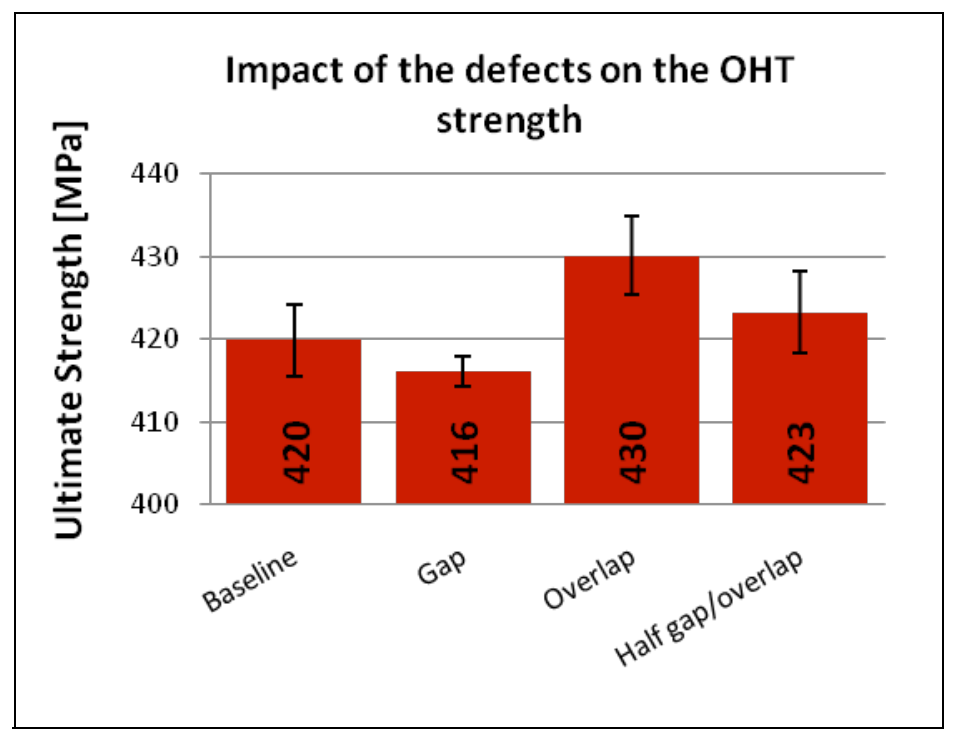

Figure 12: OHT strength variation as a function of the type of defect 


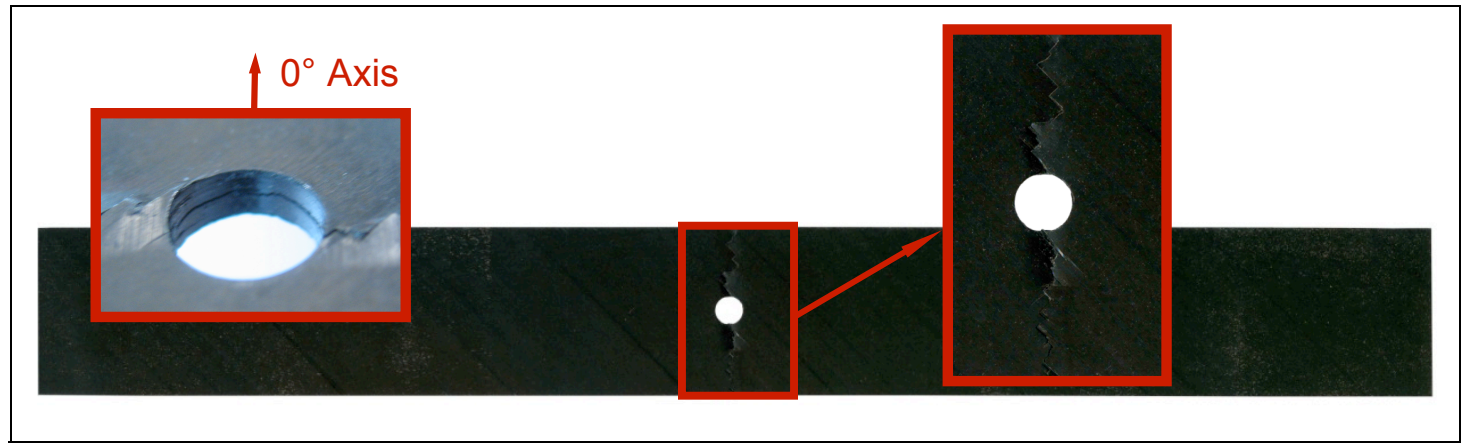

Figure 13: Typical OHC failure for a coupon without defect

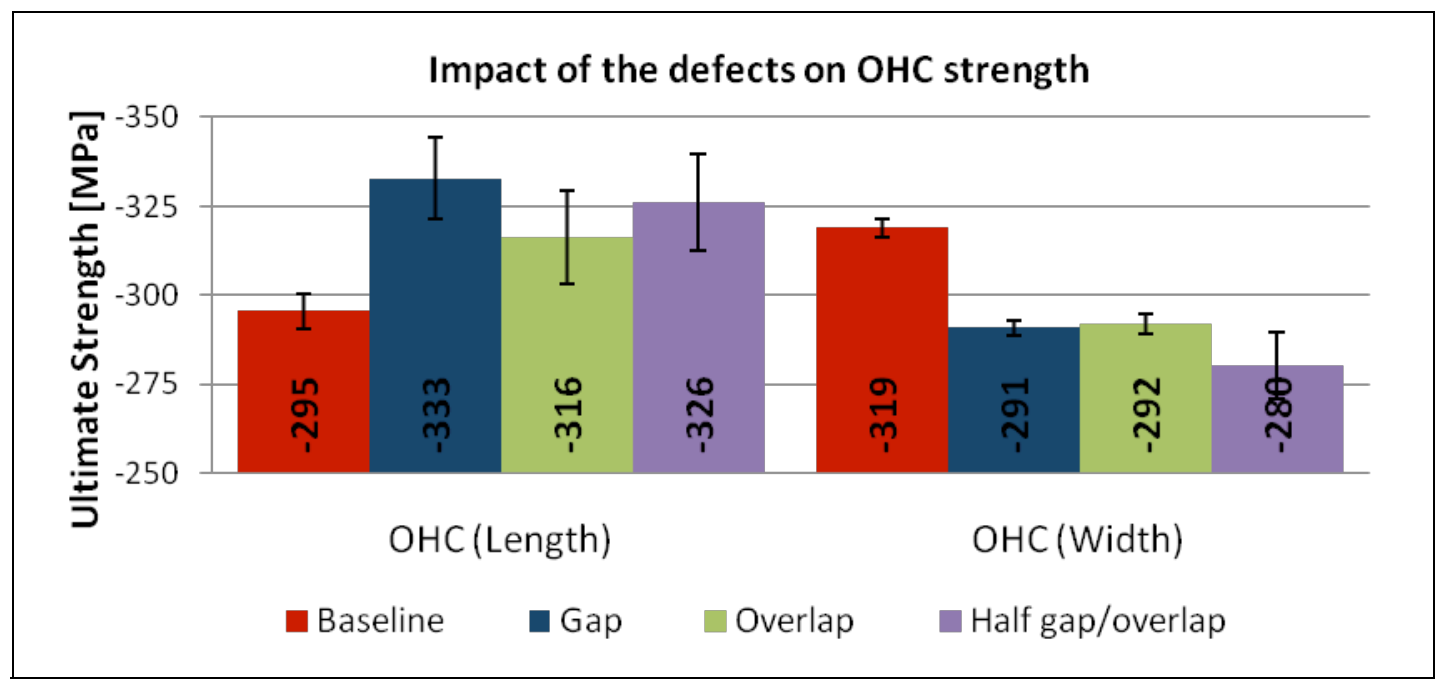

Figure 14: OHC strength variation as a function of the type of defect 


\section{Tables}

\begin{tabular}{|c|c|c|c|c|c|}
\hline & & Gap & Overlap & $\begin{array}{c}\text { Half } \\
\text { Gap/Overlap }\end{array}$ & Twisted Tow \\
\hline \multicolumn{2}{|c|}{ Tension } & - & - & & 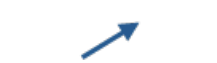 \\
\hline \multicolumn{2}{|c|}{ Compression } & - & & - & - \\
\hline \multirow{2}{*}{$\begin{array}{c}\text { In-Plane } \\
\text { Shear }\end{array}$} & Length & & $\longrightarrow$ & 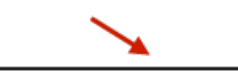 & $\Delta$ \\
\hline & Width & & $\Delta$ & 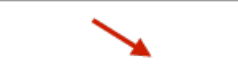 & 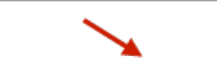 \\
\hline \multicolumn{2}{|c|}{$\mathrm{OHT}$} & 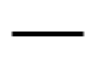 & $\longrightarrow$ & $\longrightarrow$ & \\
\hline \multirow{2}{*}{$\mathrm{OHC}$} & Length & & & & \\
\hline & Width & $y$ & $x$ & $y$ & \\
\hline \multicolumn{6}{|c|}{$\geq 3 \%$ increase (up to $13 \%$ ) } \\
\hline
\end{tabular}

Table 1: Performance comparison of the different defect configurations 